\title{
Drug utilization pattern of acute poisoning admissions in medicine intensive care unit
}

\author{
Siddharth Athawale ${ }^{1, *}$, M. D. Kulkarni' ${ }^{2}$, S. M. Doifode ${ }^{3}$ \\ ${ }^{1}$ PG Student, ${ }^{2}$ Associate Professor, ${ }^{3}$ Professor \& HOD, Dept. of Pharmacology, Government Medical College and Hopsital, \\ Aurangabad, Maharashtra, India
}

*Corresponding Author:

Email: siddharthathawale20@gmail.com

\begin{abstract}
Introduction: Acute poisoning is a very common \& life threatening crisis and leads to serious conditions and even death of patients. Poisoning may be due to self consumption or accidental of various chemical compounds, medications and poisonous animals. The various drugs used in poisoning and there clinical outcome with different poisoning substances was investigated in this study Material and Methods: The patients which have been diagnosed with acute poisoning in Medical intensive care unit from January 2017 to July 2017 are included in this study.

Results: During 7 months of study total 154 patients of acute poisoning were included in this study. Male patients were 111 (72\%) while female patients were $43(28 \%)$. Mean age of patients was $34.97+14.46$ years. The commonest cause of poisoning was by use of pesticides followed by venomous snake bite. Drugs prescribed to patients were mainly symptomatic like H2 blockers $(99.3 \%)$, antiemetics (97.4\%), and antimicrobials (90.2\%). Specific antidotes prescribed were Atropine (92.8\%), pralidoxime $(79.8 \%)$ and anti-snake venom $(17.5 \%)$

Conclusions: Organophosphorus compounds were the most common among poisons, followed by Snake bite. Gastroprotective and antiemetic drugs are most commonly prescribed drugs.
\end{abstract}

Keywords: Poisoning, Drug utilization, Intensive care unit.

\section{Introduction}

Poisoning is defined as the occurrence of harmful effects resulting from exposure to a foreign chemical or a xenobiotic, it can occur by means of ingestion, inhalation or contact; unintentionally or intentionally. ${ }^{1,2}$ Acute poisoning is a single or repeated exposure of poisoning agents for limited time duration. Poisoning may be due to self-consumption or accidental of various chemical compounds, medications and poisonous animals. ${ }^{3}$ Most common causes for emergency admissions which leads to mortality are due to acute poisoning and venomous snake bites in various nations. ${ }^{4}$ The nature of poison varies according to region and also differs in same country relating to cultural diversity and various other factors. ${ }^{5}$ Early diagnosis, treatment and prevention are the only measure through which this problem can be solved. ${ }^{6,7}$ As poisoning is very common and major public health issue the hospital services receives lot of strain and the victims have to face a great financial burden. According to the national annual report of India in 2015, death due to acute poisoning was 26173. The data showed that $39.1 \%$ of all causes of other causes of deaths in the year 2015 was due to accidental poisoning. Around $27.9 \%$ of suicidal deaths was due to poison ingestion during $2015 .^{8}$

Drug utilization studies (DUS) is "the marketing, distribution, prescription and use of drugs in a society, with special emphasis on the resulting medical, social and economic consequences". ${ }^{9}$ By use of drug utilization studies we can analyze the health care systems, also they are powerful exploratory tools to know the role of drugs in the society. They help in making decisions in socio- medical and financial basis for the health care systems. Due to various varieties of different acute diseases, which are admitted in Intensive care unit it, becomes an ideal center to study the prescription pattern of various drugs. ${ }^{4}$ The treatment received by patients of acute poisoning depends upon the availability of antidotes, essential drugs \& emergency protocols. Therefore, every institution must regularly evaluate drug utilization in different departments and their protocols to improve rationalization of drugs. ${ }^{10}$

Therefore, we designed this study to know prescription pattern, different poisoning agents and their clinical outcome in patients with acute poisoning admissions at a tertiary care teaching hospital.

\section{Materials and Methods}

A retrospective study was conducted from January 2017 to July 2017 in the Medical intensive care unit of tertiary care hospital.

\section{Study criteria \\ Inclusion criteria}

1. Patients admitted in intensive care unit with diagnosis of acute poisoning.

2. Patients of either sex

\section{Exclusion criteria}

1. Patients with incomplete case papers were excluded

2. Other types of poisoning like food \& drugs were not included

Approval from ethics committee was taken. Data collected was of age, duration in ICU, final diagnosis, drugs prescribed and clinical outcome of patients from case papers. 
The data obtained was then analyzed in Microsoft excel. Qualitative data are expressed in frequency \& percentage while quantitative data are expressed with mean \pm standard deviation $(\mathrm{SD})$

\section{Results}

Total 154 patients of acute poisoning were treated. Male patients were $111(72 \%)$ while female patients were $43(28 \%)$. Mean age of patients was $34.97+14.46$ years. Mean duration of ICU stay was $6.2 \pm 4.2$ days. (Fig.no.1) Out of 154 cases 106 were transferred to ward,
47 were expired and 1 was discharged against medical advice (DAMA) (Fig. no.2) (Tableno.1). Most common route of exposure was by ingestion of poisons $(82.4 \%)$ followed by dermal exposure $(17.5 \%)$. The commonest cause of poisoning was by use of pesticides followed by venomous snake bite. Drugs prescribed to patients were mainly symptomatic like H2 blockers (99.3\%), antiemetics (97.4\%), and antimicrobials (90.2\%) (Fig.no.3).Specific antidotes prescribed were Atropine (92.8\%), pralidoxime $(79.8 \%)$ and anti-snake venom $(17.5 \%)$

\section{Table 1: Demographic, Reason \& Outcome}

\begin{tabular}{|l|c|}
\hline Demographic & Frequency $(\%)(\mathbf{n}=\mathbf{1 5 4})$ \\
\hline Male & $111(72 \%)$ \\
\hline Female & $43(28 \%)$ \\
\hline Age (Mean+SD) & $34.97 \pm 14.46$ years \\
\hline Reason & $36(23.3 \%)$ \\
\hline Accidental & $118(76.6 \%)$ \\
\hline Suicidal & $6.2 \pm 4.2$ days \\
\hline Length of Hospital Stay (Mean+SD) & $106(69 \%)$ \\
\hline Outcome & 1 \\
\hline Transferred to ward & $47(30 \%)$ \\
\hline DAMA & \\
\hline Expired &
\end{tabular}

Table 2: Various drugs utilized

\begin{tabular}{|l|c|}
\hline Treatment & Frequency $\mathbf{( \% )}(\mathbf{n}=\mathbf{1 5 4})$ \\
\hline Atropine sulfate & $92.80 \%$ \\
\hline Pralidoxime & $79.80 \%$ \\
\hline Anti-snake venom & $17.50 \%$ \\
\hline Neostigmine & $12.30 \%$ \\
\hline Gastroprotective & $99.30 \%$ \\
\hline Antiemetics & $97.40 \%$ \\
\hline Antimicrobials & $90.20 \%$ \\
\hline Midazolam & $18.10 \%$ \\
\hline Lorazepam & $5.80 \%$ \\
\hline Vitamin K & $0.06 \%$ \\
\hline Tramadol & $5.10 \%$ \\
\hline Hydrocortisone & $13.60 \%$ \\
\hline Tetanus Toxoid & $13.60 \%$ \\
\hline Flanzen & $5.10 \%$ \\
\hline Lignocaine liquid & $1.90 \%$ \\
\hline Sucralfate syrup & $1.90 \%$ \\
\hline
\end{tabular}

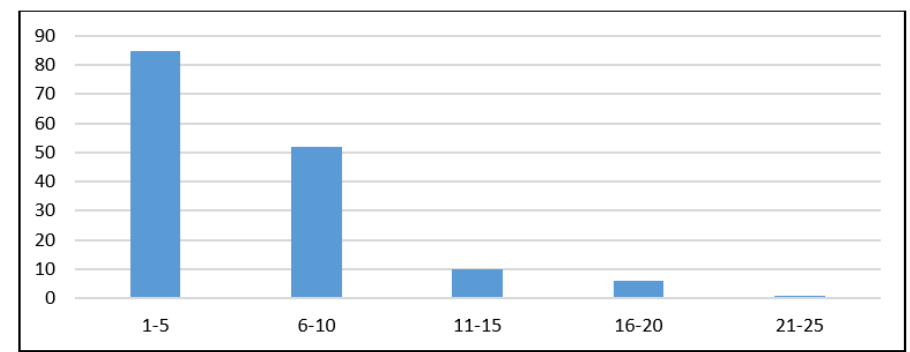

Fig. 1: Total Duration of Stay (Days) 


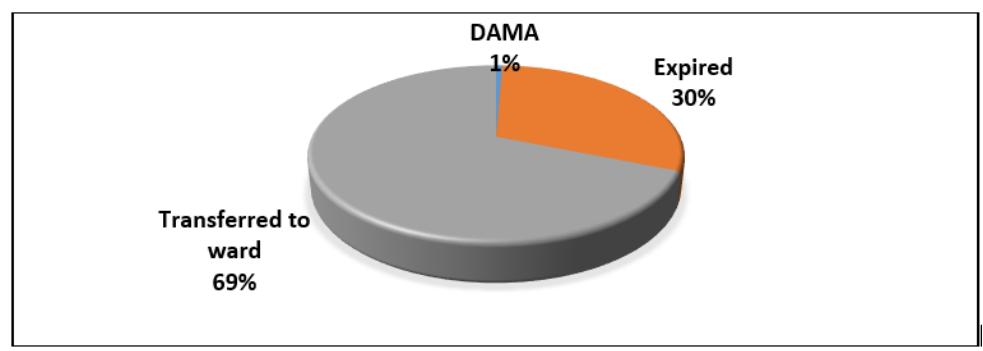

Fig. 2: Outcome in Patients

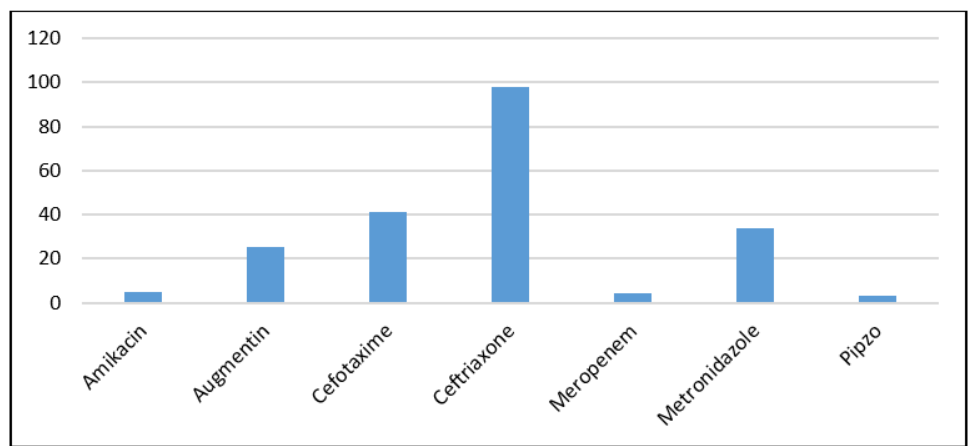

Fig. 3: Various Antibiotics utilized

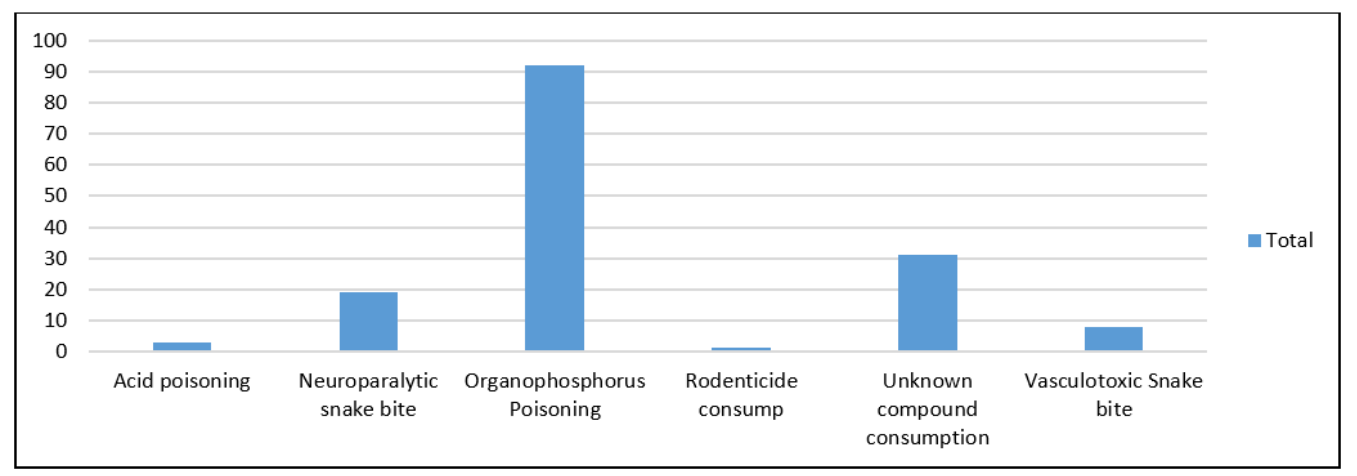

Fig. 4: Diagnosis

\section{Discussion}

Acute Poisoning is upcoming medical \& social problem now days everywhere in the world. Due to which there arises serious health issues, mortality, and burden on health services. ${ }^{2,11}$

In this study, a male predominance was observed which the similar pattern is observed by other studies in India. ${ }^{1,}{ }^{12-14}$ This trend may be due to increased occupational hazard and exposure of men to stress being the earning members compared to women. Majority of cases in this study are aged between $15-50$ years, which is similar to the other Indian studies, and the reasons behind are mostly due to domestic, educational and employment related stress. Patients who consumed the poisoned with suicidal intent were $77 \%$ and those who were exposed accidentally were $23 \% .^{4}$

Poisoning and Snake bite persist to stay a major health problem in the current study. The most common type of poisoning was by consumption of pesticides, as they are readily available among farmers and households. After pesticides poisoning, snake bite is most common by both neurotoxic as well as vasculotoxic snakes. ${ }^{15}$ Organophosphorus compounds are common among the pesticides and their treatment included mainly Atropine and Oximes. ${ }^{16,17}$

Snake bite is a very serious but neglected public issue in various countries which affect mainly the rural population. In India, snake bites take a heavy toll of human lives, and therefore warrant urgent attention. Snake bite cases accounted for $17.5 \%$ of the present study population. In the present study, polyvalent antisnake venom (ASV) was administered in all cases of snake poisoning. Neostigmine with alternate doses of Atropine were administered IV to improve respiratory muscles weakness in neurotoxic snake bites. In addition, Tetanus toxoid injection was given to $13.6 \%$ along with Tramadol and Serratiopeptidase.

The Symptomatic treatment mainly involved usage of Gastroprotective agents like $\mathrm{H}_{2}$ blockers, antiemetic which were observed in other studies too. ${ }^{10,} 18$ 
Antimicrobials were prescribed to $90.2 \%$ patients and most used were Cephalosporins, followed by Metronidazole. ${ }^{19,20}$ In intensive care unit broad spectrum antibiotics are given as empirical therapy. Over estimation of the severity of illness may be the main reason for such an empirical use of antimicrobials in intensive care unit.

\section{Conclusion}

Organophosphorus compounds were the most common among poisons, followed by Snake bite. Gastroprotective, antiemetic drugs, while antidotes like Atropine, PRAM and ASV are most commonly prescribed drugs. More studies should be encouraged in different hospital settings and geographical areas to study the pattern of poisoning, its morbidity, and mortality. A long-term prospective study is needed in case of poisoning for understanding the clinical pattern and management techniques, which can help in intervention for a better outcome. Establishment of a poison center within the region also will facilitate in preventing and controlling such poisoning events.

\section{References}

1. Aravind A, Rai M. Pattern of Acute Poisoning Admissions in the Medical Intensive Care Unit of a Tertiary Care Hospital. Int J Pharm Sci Drug Res. 2014;6(3):239-42.

2. Prashant Gupta, Anil Kumar SPS. Pattern Of Cases Of Acute Poisoning In A Rural. Natl J Community Med. 2016;7(4):4-7.

3. Cahfer Guloglu IHK. Acute poisoning cases admitted to a university hospital emergency department in Diyarbakir, Turkey. Hum Exp Toxicol. 2005;24(February):1-6.

4. Simon A, Anagha R, Anns J. Drug Utilization Study And Evaluation Of Rational Use Of Medicines In The Medical Emergency Poison Department And Intensive Care Unit Of A Government Hospital ; A Prospective Study. WORLD J Pharm Pharm Sci. 2018;7(1):1169-89.

5. Ramesha KN, Rao KBH, Kumar GS. Pattern and outcome of acute poisoning cases in a tertiary care hospital in Karnataka , India. Indian J Crit Care Med. 13(3):152-5.

6. Ahuja H, Mathai AS, Pannu A, Arora R. Acute Poisonings Admitted to a Tertiary Level Intensive Care Unit in Northern India : Patient Profile and Outcomes. J Clin Diagnostic Res. 2015;9(10):1-4.

7. Acharya S, Lakshminarayana K. Assessment of Poisoning Cases in a Tertiary Care Hospital. Int J Biomed Res. 2014;5(9):578-81.

8. Ministry of Home Affairs Government of India. National Crime Records Bureau Ministry of Home Affairs Government of India. Page no. 8, Page no.205.

9. Trykk LH, Aksidenstrykkeri N, Isbn AS. Introduction to Drug Utilization Research Introduction to Drug Utilization Research. 2003. page no. 9.

10. Asari PD, Shah SM, Amin AJ, Patel ND. Drug Utilization Study on Acute Poisoning Cases Treated at a Tertiary Care Hospital in Western Part of India. ASIA PACIFIC J Med Toxicol. 2016;5(December 2015):20-4

11. Maharani B, Vijayakumari N. Profile of poisoning cases in a Tertiary care Hospital , Tamil Nadu. J Appl Pharm Sci. 2013;3(1):91-4.

12. Patil A, Peddawad R, Chandra V, Verma S, Gandhi H.
Profile of Acute Poisoning Cases Treated in a Tertiary Care Hospital : a Study in Navi Mumbai. ASIA PACIFIC J Med Toxicol. 2014;3(March):36-40.

13. Irteqa Ali, Kamal kumar Sawlani, D Himanshu CC, Et.al. Study Of Pattern And Outcome Of Acute Poisoning Cases At Tertiary Care. J Evid Based Med Heal. 2017;4(6):326-31.

14. Rr S, Kumar A, Uraiya D, Dhaon P, Singh RR, Kumar A, et al. Retrospective analysis of poisoning cases admitted in a tertiary care hospital in North Eastern UP , India. Int J Med Res Rev. 2016;4(7):1172-7.

15. Chavan VR, Arshad M, Raghunandan M. A Study On Pattern Of Acute Poisoning In An Emergency Department Of A Tertiary Care Hospital. Asian J Pharm Clin Res. 2016;9(3):8-10.

16. Sunaina Godhwani, Sumeet Godhwani KLT. Management Of Organic Insecticide Poisoning In Intensive Care Unit ( I . C . U .). Indian J Anaesth. 2004;48(4):4-7.

17. Sungur M, Güven M. Intensive care management of organophosphate insecticide. Crit Care. 2001;5(4):3-7.

18. Aravinda V, Bedwal A, Rajarathna K, M NS. Pattern and pharmacotherapy of acute poisonings presenting to a tertiary care centre. Int J Basic Clin Pharmacol. 2017;6(4):879-86.

19. Raut A, Pawar A, Shaj K, Dave P. Treatment Approaches for Management of Poisonings in. Ann Public Heal Res. 2017;4(4):1-7.

20. Khan PA, M DC, Kumar MM, Nousheen BBS. A Study On Treatment Pattern And Outcomes Of Poisoning Cases In A Tertiary Care And Government District Hospital. Int J Pharm Pharm Sci. 2017;9(4). 